\title{
Observer Design for the Doyle-Fuller-Newman Li-ion Battery Model Without Electrolyte Dynamics
}

\author{
R. Drummond and S. R. Duncan
}

\begin{abstract}
An observer is designed for the DoyleFuller-Newman electrochemical model of a Li-ion battery under the assumption of constant ion exchange current density and MacInnes' equation being concentration independent. For this nonlinear system, the observer is shown to experience both exponential convergence and a degree of robustness. This result extends the class of electrochemical model for battery observer design, generating improved estimates for the state-ofcharge and for the spatial variation of the overpotential across an electrode.
\end{abstract}

Index Terms - State-of-charge estimation, Li-ion batteries, electrochemical models, observer design.

\section{INTRODUCTION}

State estimation of a Doyle-Fuller-Newman type Liion battery model with an observer is considered. Using current and voltage information, the observer is shown to achieve exponential convergence in its estimate of the model states, with this exponential convergence holding robustly even when there is a mismatch between the plant and the observer model. A main feature of the observer is that it does not require the inversion of the open circuit voltage (OCV), with the flatness of this curve limiting the observability of existing observer designs [3, 25]. The observer design allows the estimated states to vary across the electrode, providing a more realistic representation of the internal state of the battery and improved estimates of the spatial distributions of the overpotential and the state-of-charge. This information is particularly useful for optimal charging, as state constraints on the overpotential are key to mitigating battery degradation and increasing capacity limits [31].

Interest in state observers for Li-ion batteries was motivated by the state-of-charge $(S o C)$ estimation problem $[33,46,21]$, where an accurate estimates of the remaining charge that can be drawn from the battery using only sensor information is desired. Along with the state-ofhealth estimation problem, which is concerned with determining battery degradation, accurate $S o C$ estimation has long been recognised as being of critical importance for the effective use of battery management systems (BMS). The practical significance of this problem, as well as its relative complexity [47], has led to a considerable amount

R. Drummond and S. R. Duncan are with the Department of Engineering Science, University of Oxford, 17 Parks Road, OX1 3PJ Oxford, United Kingdom, email \{ross.drummond,stephen.duncan\}@eng.ox.ac.uk. Work supported by the Engineering and Physical Sciences Research Council through grant EP/P005411/1-"Structured electrodes for improved energy storage". of interest in its solution, with the proposed methods being cross-disciplinary, combining techniques from battery modelling, control systems and information science. One such solution method involves the design of observers and it is this approach that is considered in this paper.

An observer is a model of a system that is able to correct for inconsistencies in its predicted internal state by using sensor information. The underlying battery model is key to the successful use of an observer, with the two main model classes being equivalent circuits [17] and electrochemical models [27]. Equivalent circuits map current to voltage using a circuit containing resistors and capacitors. This approach has the benefit of being fast, relatively simple and readily parametrisable. For these reasons, equivalent circuits have become the most popular form of model for battery observer design, see for instance [6]. However, from the perspective of gaining insight into the internal electrochemical state of the battery, a fundamental problem follows from the use of such circuits within an observer, in the sense that their internal states lack a direct physical interpretation. Several recent studies, such as [45], have investigated the physical interpretations of such circuits, with the approach of [14] using realisation theory to synthesise circuits from the impedance function of an electrochemical model, but such interpretations are still far from definitive.

The other popular model class for batteries are electrochemical. These models describe phenomena including lithium intercalation in the particles and charge conservation, typically using a mixture of partial differential and algebraic equations. An understanding of the battery electrochemistry is built into the foundations of these models, and as a result, their internal model states typically relate to a physical variable of interest within the battery, such as ionic concentration within the particles. Obtaining more accurate estimates of these internal variables provides a richer information set for the battery management system to control. The trade-off involved with using electrochemical models lies in their complexity, both in terms of their analysis and in their simulation speed. For this reason, model order reduction methods [39] or simplifying assumptions on the electrochemistry $[28,37,22,24]$ are often applied with them, resulting in a loss in their electrochemical interpretation, which undermines these models' strongest attribute.

A benchmark electrochemical model is the Doyle-FullerNewman (DFN) model, otherwise known as the P2D model, which was developed in [10, 42] and explained in detail in [27]. This is the model considered in this 
paper. Existing results on the state-of-charge estimation using observers with electrochemical models include [32, $25,26,24,9,7,48,6]$ and typically use variations of the single particle model (SPM), with the results of [9] being closest in spirit to those presented here, which are also derived from a robust stability perspective. These previous results on $S o C$ estimation with electrochemical models have revealed fundamental issues related to observability, due to the flatness of the OCV curve [25, 47], whose inversion was tackled in [3] using polynomial optimisation. Recently, to avoid this voltage observability issue, the use of additional sensor measurements, such as strain induced swelling [29, 36] or temperature [41], has been proposed.

The results of this paper present a different approach by designing the observer for the nonlinear partial differential equations of a model derived from the DFN model, but with fewer assumptions on the electrochemistry than the single particle model. The model is obtained by including double layer effects (allowing a state-space form to be obtained) and by assuming that the ionic exchange current and MacInnes' equation are concentration independent. This allows accurate estimates of the particle surface concentration and the overpotential across an electrode to be obtained. Furthermore, since only open circuit potentials, not the open circuit voltage, are used in the battery model, the (often non-invertible) OCV does not have to be inverted with the proposed approach. It was also found that considering the observer design in the PDE setting provided insight into the correct structure that the observer should take, with problems associated with the design in the finite dimensional setting also being discussed in Section IV. These results demonstrate how the DFN model can be interpreted using systems analysis for potentially other applications, such as optimal control and parameter estimation.

\section{Doyle-Fuller-Newman Model}

The observers used in this paper are designed for a form of the classical Doyle-Fuller-Newman electrochemical Liion battery model with double layer capacitance effects included, as in $[11,10,27]$. The model is "pseudo-two dimensional", with a spatial co-ordinate $x$ spanning the battery length, from one current collector to the other and crossing both electrodes and the separator, and with another co-ordinate $r$ pointing normally outwards in a disk from each point $x$ in the electrodes, with $r$ defining the domain of the electrode particles. The parameters of the model are given in Table I and its variables are given in Table II. The spatio-temporal variables of the model are the concentration of ions in the electrolyte $c_{e}(x, t)$, the scaled ionic concentration in the solid particles $u_{s}(x, r, t)$ and its surface value $u_{s}^{\text {surf }}(x, t)=u_{s}\left(x, R_{s}, t\right)$, the current density $i_{s}(x, t)$ and potential $\phi_{e}(x, t)$ in the electrolyte and, correspondingly, in the solid phase $i_{s}(x, t), \phi_{s}(x, t)$, the applied current density $i(t)$ and the measured voltage $v(t)$. For the sake of clarity, subscripts detailing the domain (cathode, anode or separator) of the model variables are

\begin{tabular}{|c|c|}
\hline \multicolumn{2}{|r|}{ Parameters } \\
\hline$c_{s}^{\max }$ & Maximum lithium concentration in the particles. \\
\hline$D_{s}$ & Diffusion coefficient in the particles. \\
\hline$R_{s}$ & Spherical radius of the particles. \\
\hline$L_{m}$ & Position in $x . \boldsymbol{m} \in\{12,23\}$ indicates the boundary. \\
\hline$L_{\text {bat }}$ & Total length of the battery. \\
\hline$\epsilon_{\mathrm{e}}$ & Porosity coefficient. \\
\hline$b$ & Bruggeman coefficient. \\
\hline$D_{\mathrm{e}}^{\mathrm{eff}}$ & Effective electrolyte diffusion coefficient. \\
\hline$a_{s}$ & Specific interfacial area. \\
\hline$t_{+}$ & Transference number. \\
\hline $\mathcal{F}$ & Faraday's constant. \\
\hline$\alpha_{a} / \alpha_{c}$ & Anode and Cathode Charge Transfer coefs. \\
\hline$R$ & Universal gas constant. \\
\hline$T$ & Temperature. \\
\hline$k$ & Exchange Rate Parameter. \\
\hline$\sigma^{\text {eff }}$ & Effective electrode conductivity. \\
\hline$\kappa^{\mathrm{eff}}$ & Effective electrolyte conductivity. \\
\hline$c_{T} \bar{V}_{0}$ & Solute Volume effect factor. \\
\hline$\chi$ & Thermodynamic factor. \\
\hline$R_{c t c}$ & Contact resistance. \\
\hline$K$ & A coefficient. $K=\frac{2\left(1-t_{+}\right) R T}{\mathcal{F}} \frac{\chi}{c_{T} \bar{V}_{0}}$ \\
\hline$R_{i}$ & $\begin{array}{l}\text { Resistance. } \\
R_{i}=\left(\frac{1}{\kappa_{1}^{\text {eff }}\left(\kappa_{1}^{\text {eff }}+\sigma_{1}^{\text {eff }}\right)}+\frac{1}{\kappa_{3}^{\text {eff }}\left(\kappa_{3}^{\text {eff }}+\sigma_{3}^{\text {eff }}\right)}+\frac{1}{\kappa_{2}^{\text {eff }}}-R_{\text {ctc }}\right)\end{array}$ \\
\hline \multicolumn{2}{|r|}{ Sub and super scripts } \\
\hline 1 & Denotes anode parameters. \\
\hline 2 & Denotes separator parameters. \\
\hline 3 & Denotes cathode parameters. \\
\hline 12 & Anode/separator boundary. \\
\hline 23 & Cathode/separator boundary. \\
\hline
\end{tabular}

Table I: Parameters of the battery model.

removed from the equations except when such labels are essential.

The following set of parabolic and algebraic partial differential equations characterise the DFN model for each electrode with it being assumed that the electrolyte's diffusion coefficient and conductivity are independent of $c_{e}(x, t)$. The electrode equations are:

- Lithium intercalation in the particles

$$
\frac{\partial u_{s}(x, r, t)}{\partial t}=D_{s} \frac{\partial^{2} u_{s}(x, r, t)}{\partial r^{2}}
$$

- Ion transport in the electrolyte

$$
\left.\epsilon_{\mathrm{e}} \frac{\partial c_{\mathrm{e}}(x, t)}{\partial t}=\epsilon_{\mathrm{e}} D_{\mathrm{e}}^{\mathrm{eff}} \frac{\partial^{2} c_{\mathrm{e}}}{\partial x^{2}}+a_{\mathrm{s}}\left(1-t_{+}\right) j(x, t)\right) .
$$

- Butler-Volmer equation

$$
j\left(\eta(x, t), i_{0}(x, t)\right)=\frac{i_{0}(x, t)}{\mathcal{F}}\left[e^{\left(\frac{\alpha_{\mathrm{a}} \mathcal{F}}{R T} \eta(x, t)\right)}-e^{\left(\frac{-\alpha_{\mathrm{C}} \mathcal{F}}{R T} \eta(x, t)\right)}\right] .
$$

- Ionic exchange current density

$$
\begin{aligned}
i_{0}(x, t) & =k \mathcal{F}\left(c_{\mathrm{s}}^{\max }-c_{\mathrm{s}}^{\text {surf }}(x, t)\right)^{\alpha_{\mathrm{a}}} \\
& \times\left(c_{\mathrm{s}}^{\text {surf }}(x, t)\right)^{\alpha_{\mathrm{c}}}\left(c_{\mathrm{e}}(x, t)\right)^{\alpha_{\mathrm{a}}} .
\end{aligned}
$$




\begin{tabular}{c|l}
\hline \multicolumn{2}{c}{ Spatial Variables } \\
\hline$r$ & $\begin{array}{l}\text { Spatial variable across the battery. } \\
\text { Spatial variable in the particles. }\end{array}$ \\
\hline \multicolumn{2}{c}{ Spatio-Temporal Variables of the DFN Model } \\
\hline$u_{s}(x, r, t)$ & $\begin{array}{l}\text { Transformed particle concentration. } \\
u_{s}^{\text {surf }}(x, t)\end{array}$ \\
& $\begin{array}{l}\text { Transformed particle surface concentration. } \\
u_{s}^{\text {surf }}(x, t)=u_{s}(x, R, t) .\end{array}$ \\
$\phi_{d l}(x, t)$ & Electrode potential. \\
& $\phi_{d l}(x, t)=\phi_{s}(x, t)-\phi_{e}(x, t)$. \\
$j(x, t)$ & Reaction rate. \\
$\eta(x, t)$ & Overpotential. \\
$U\left(u_{s}^{\text {surf }}(x, t)\right)$ & Open circuit potential. \\
$i(t)$ & Applied current density. \\
$v(t)$ & Measured voltage \\
\hline \multicolumn{2}{c}{ Algebraic Variables of the DFN Model } \\
\hline$c_{e}(x, t)$ & Concentration of lithium in the electrolyte. \\
$\phi_{s}(x, t)$ & Electric potential in the solid phase. \\
$\phi_{e}(x, t)$ & Electric potential in the electrolyte. \\
$i_{s}(x, t)$ & Current density in the solid phase. \\
$i_{e}(x, t)$ & Current density in the electrolyte. \\
\hline
\end{tabular}

Table II: Spatio-temporal variables of the DFN model.

\section{- Overpotential}

$$
\eta(x, t)=\phi_{\mathrm{s}}(x, t)-\phi_{\mathrm{e}}(x, t)-U\left(u_{\mathrm{s}}^{\mathrm{surf}}(x, t)\right) .
$$

- Ohm's law

$$
\frac{i_{\mathrm{s}}(x, t)}{\sigma^{\mathrm{eff}}}=-\frac{\partial \phi_{\mathrm{s}}(x, t)}{\partial x} .
$$

- MacInnes' equation

$$
\begin{aligned}
\frac{i_{\mathrm{e}}(x, t)}{\kappa^{\mathrm{eff}}}= & -\frac{\partial \phi_{\mathrm{e}}(x, t)}{\partial x} \\
& +\frac{2\left(1-t_{+}\right) R T}{\mathcal{F}} \frac{\chi}{c_{\mathrm{T}} \bar{V}_{0}} \frac{\partial \ln c_{\mathrm{e}}(x, t)}{\partial x} .
\end{aligned}
$$

- Kirchoff's law

$$
i_{\mathrm{s}}(x, t)+i_{\mathrm{e}}(x, t)=i_{\mathrm{app}}
$$

- Divergence equation

$$
\frac{\partial i_{\mathrm{e}}(x, t)}{\partial x}=a_{\mathrm{s}} \mathcal{F} j\left(\eta, i_{0}\right) .
$$

In the separator, only unforced electrolyte diffusion occurs

$$
\frac{\partial c_{\mathrm{e}}(x, t)}{\partial t}=D_{\mathrm{e}}^{\mathrm{eff}} \frac{\partial^{2} c_{\mathrm{e}}}{\partial x^{2}}
$$

The complexity of these equations has made the analysis of this model challenging from a systems perspective [34], even though it is considered a benchmark Li-ion battery model [35]. In this paper, the recent formulation of the DFN model presented in [11] is exploited to describe the model in a state-space form.

It was shown in [11] that by including fast double layer capacitance effects into the DFN model, using the method introduced by Ong and Newman [30], a state-space formulation could be obtained with all the algebraic variables being eliminated. With this method, double layer effects are included by augmenting the divergence equation of
(9) to account for the dynamics of the electrode potential $\phi_{d l}(x, t)=\phi_{s}(x, t)-\phi_{e}(x, t)$

$$
a C \frac{\partial \phi_{d l}(x, t)}{\partial t}=\frac{\partial i_{e}(x, t)}{\partial x}-a_{s} F j\left(\eta, i_{0}\right),
$$

so that, after further manipulations of MacInnes' equation and Kirchoff's and Ohm's laws (described in [11]), the DFN model can then be expressed as the following statespace system

$$
\begin{aligned}
\epsilon_{e} \frac{\partial c_{e}(x, t)}{\partial t} & =\epsilon_{e} D_{e}^{\mathrm{eff}} \frac{\partial^{2} c_{e}(x, t)}{\partial x^{2}}+a_{\mathrm{s}}\left(1-t_{+}\right) j\left(\eta, i_{0}\right) \\
\frac{\partial u_{s}(r, t)}{\partial t} & =D_{s} \frac{\partial^{2} u_{s}(r, t)}{\partial r^{2}} \\
a C \frac{\partial \phi_{d l}(x, t)}{\partial t} & =\left(\frac{\kappa^{\mathrm{eff}} \sigma^{\mathrm{eff}}}{\sigma^{\mathrm{eff}}+\kappa^{\mathrm{eff}}}\right) \frac{\partial^{2} \phi_{d l}(x, t)}{\partial x^{2}} \\
& -a_{s} F j\left(\eta, i_{0}\right)+\left(\frac{K \sigma^{\mathrm{eff}}}{\sigma^{\mathrm{eff}}+\kappa^{\mathrm{eff}}}\right) \frac{\partial^{2}}{\partial x^{2}} \ln (c(x, t))
\end{aligned}
$$

This system has a state-space of $\left\{c_{e}, u_{s}, \phi_{d l}\right\}$ and does not require the use of algebraic variables such as $i_{e}(x, t)$ to be solved forward in time. Even though this system is nonlinear, it can be analysed in terms of feedback loops involving monotonic nonlinear functions [19], a perspective which is exploited here for the observer design problem.

With these dynamics, the states satisfy the boundary conditions of Table III and the voltage can be expressed as

$$
\begin{aligned}
& v(t)=-\left(\frac{\kappa_{1}^{\mathrm{eff}}}{\kappa_{1}^{\mathrm{eff}}+\sigma_{1}^{\mathrm{eff}}}\right) \phi_{d l}(0, t)-\left(\frac{\sigma_{1}^{\mathrm{eff}}}{\kappa_{1}^{\mathrm{eff}}+\sigma_{1}^{\mathrm{eff}}}\right) \phi_{d l}\left(L_{12}, t\right) \\
& +\left(\frac{\sigma_{3}^{\mathrm{eff}}}{\kappa_{3}^{\mathrm{eff}}+\sigma_{3}^{\mathrm{eff}}}\right) \phi_{d l}\left(L_{23}, t\right)+\left(\frac{\kappa_{3}^{\mathrm{eff}}}{\kappa_{3}^{\mathrm{eff}}+\sigma_{3}^{\mathrm{eff}}}\right) \phi_{d l}\left(L_{\mathrm{bat}}, t\right) \\
& -R_{i} i(t) \\
& -K\left(\left(\frac{\sigma_{1}^{\mathrm{eff}}}{\kappa_{1}^{\mathrm{eff}}\left(\kappa_{1}^{\mathrm{eff}}+\sigma_{1}^{\mathrm{eff}}\right)}-1\right)\left(\ln c_{e}\left(L_{12}, t\right)-\ln c_{e}(0, t)\right)\right. \\
& -\frac{1}{\kappa_{2}^{\mathrm{eff}}}\left(\ln c_{e}\left(L_{23}, t\right)-\ln c_{e}\left(L_{12}, t\right)\right) \\
& \left.-\left(\frac{\sigma_{3}^{\mathrm{eff}}}{\kappa_{3}^{\mathrm{eff}}\left(\kappa_{3} \mathrm{eff}+\sigma_{3}^{\mathrm{eff}}\right)}-1\right)\left(\ln c_{e}\left(L_{\mathrm{bat}}, t\right)-\ln c_{e}\left(L_{23}, t\right)\right)\right)
\end{aligned}
$$

with the resistance $R_{i}$ defined in Table I. The subscripts 1,2 and 3 denote the region in the battery for the parameter, with the subscript 1 implying the anode, 2 being the separator and 3 the cathode: for example in this notation $\kappa_{1}^{\text {eff }}$ is the value of the electrolyte conductivity in the anode. Similarly, the position $x=L_{12}$ is the boundary between the anode and separator and $x=L_{23}$ is the boundary between the separator and the cathode.

Since the time constants of the double layer are an order of magnitude faster than those of lithium intercalation within the particles, then, for low C-rate charging currents, the inclusion of double layer capacitance effects can often have a negligible impact upon the overall solution accuracy of the model. However, from the perspective of systems analysis, the ability to obtain a state-space formulation 


\begin{tabular}{c|c}
\hline \hline \multicolumn{2}{c}{ Boundary conditions of the DFN Model } \\
\hline \hline Current collector/electrode & \multicolumn{1}{c}{ Electrode/separator } \\
\hline$\frac{\partial c(x, t)}{\partial x}=0$ & $\left.D_{e}^{\text {eff }} \frac{\partial c(x, t)}{\partial x}\right|_{\text {elec }}=\left.D_{e}^{\text {eff }} \frac{\partial c(x, t)}{\partial x}\right|_{s e p}$ \\
\hline$\frac{\partial \phi_{d l}(x, t)}{\partial x}=-\frac{i(t)}{\sigma^{\text {eff }}}$ & $\frac{\partial \phi_{d l}(x, t)}{\partial x}=\frac{i(t)}{\kappa^{\text {eff }}}+\frac{K}{\kappa^{\text {eff }}} \frac{\partial \ln c_{e}(x, t)}{\partial x}$ \\
\hline Particle centre & \multicolumn{1}{c}{ Particle surface } \\
\hline$u_{s}(x, 0, t)=0$ & $\left.\frac{1}{R_{s}} \frac{\partial u_{s}}{\partial r}\right|_{R_{s}}-\frac{u_{s}\left(x, R_{s}, t\right)}{R_{s}^{2}}=\frac{-j(\eta)}{D_{s}}$ \\
\hline
\end{tabular}

Table III: Boundary conditions for the DFN model with double layer capacitance. The subscript sep indicates a derivative pointing into the separator while the subscript elec indicates a derivative pointing into the electrode.

\begin{tabular}{c|c}
\hline \hline \multicolumn{2}{c}{ Boundary conditions of the reduced DFN Model } \\
\hline \hline Current collector/electrode & Electrode/separator \\
\hline$\frac{\partial \phi_{d l}(x, t)}{\partial x}=-\frac{i(t)}{\sigma^{\text {eff }}}$ & $\frac{\partial \phi_{d l}(x, t)}{\partial x}=\frac{i(t)}{\kappa^{\text {eff }}}$ \\
\hline Particle centre & \multicolumn{2}{|c}{ Particle surface } \\
\hline$u_{s}(x, 0, t)=0$ & $\left.\frac{1}{R_{s}} \frac{\partial u_{s}}{\partial r}\right|_{R_{s}}-\frac{u_{s}\left(x, R_{s}, t\right)}{R_{s}^{2}}=\frac{-j(\eta)}{D_{s}}$ \\
\hline
\end{tabular}

Table IV: Boundary conditions for the reduced DFN model with double layer capacitance.

of the model with a feedback interpretation, justifies the inclusion of this additional effect. It is emphasised that the transformation of the DFN model into this statespace form was achieved through the addition of neglected dynamics (double layer effects), not by simplifications in the electrochemistry.

\section{Model Assumptions}

In this section, the following assumptions are applied to the DFN model (12) to make the observer design problem feasible.

1. The ion exchange current density is constant across an electrode $i_{0}(x, t)=i_{0}$.

2. The applied current $I(t)$ is known.

3. MacInnes' equation is concentration independent.

Assumption 1 is applied to facilitate the observer design as it makes the nonlinearities monotonic, but the simulations of Section IV are applied with a DFN model plant with $i_{0}(x, t)$ as defined in (4). Assumption 3 is perhaps the least electrochemically justified condition (although is often used in electrochemical modelling as it implies equal transference numbers as used as in [44] for instance), but greatly simplifies the analysis as it decouples the equation system. Under this assumption, the current density in the electrolyte can be expressed as

$$
i_{e}(x, t)=-\kappa^{\mathrm{eff}} \frac{\partial \phi_{e}(x, t)}{\partial x} .
$$

This allows the logarithmic term from the model equations to be eliminated, with the dynamical equations becoming

$$
\begin{aligned}
\epsilon_{e} \frac{\partial c_{e}(x, t)}{\partial t} & =\epsilon_{e} D_{e}^{\mathrm{eff}} \frac{\partial^{2} c_{e}(x, t)}{\partial x^{2}}+a_{\mathrm{s}}\left(1-t_{+}\right) j(\eta) \\
\frac{\partial u_{s}(r, t)}{\partial t} & =D_{s} \frac{\partial^{2} u_{s}(r, t)}{\partial r^{2}}, \\
a C \frac{\partial \phi_{d l}(x, t)}{\partial t} & =\left(\frac{\kappa^{\mathrm{eff}} \sigma^{\mathrm{eff}}}{\sigma^{\mathrm{eff}}+\kappa^{\mathrm{eff}}}\right) \frac{\partial^{2} \phi_{d l}(x, t)}{\partial x^{2}}-a_{s} F j(\eta),
\end{aligned}
$$

and the voltage expression becoming

$$
\begin{aligned}
v(t) & =-\left(\frac{\kappa_{1}^{\mathrm{eff}}}{\kappa_{1}^{\mathrm{eff}}+\sigma_{1}^{\mathrm{eff}}}\right) \phi_{d l}(0, t)-\left(\frac{\sigma_{1}^{\mathrm{eff}}}{\kappa_{1}^{\mathrm{eff}}+\sigma_{1}^{\mathrm{eff}}}\right) \phi_{d l}\left(L_{12}, t\right) \\
& +\left(\frac{\sigma_{3}^{\mathrm{eff}}}{\kappa_{3}^{\mathrm{eff}}+\sigma_{3}^{\mathrm{eff}}}\right) \phi_{d l}\left(L_{23}, t\right)+\left(\frac{\kappa_{3}^{\mathrm{eff}}}{\kappa_{3}^{\mathrm{eff}}+\sigma_{3}^{\mathrm{eff}}}\right) \phi_{d l}\left(L_{\mathrm{bat}}, t\right) \\
& -R_{i} i(t) .
\end{aligned}
$$

Under these assumptions, the electrolyte concentration $c_{e}$ becomes an unobservable state with regards to the voltage and so the two state system

$$
\begin{aligned}
\frac{\partial u_{s}(r, t)}{\partial t} & =D_{s} \frac{\partial^{2} u_{s}(r, t)}{\partial r^{2}} \\
\frac{\partial \phi_{d l}(x, t)}{\partial t} & =\left(\frac{\kappa^{\mathrm{eff}} \sigma^{\mathrm{eff}}}{a C\left(\sigma^{\mathrm{eff}}+\kappa^{\mathrm{eff}}\right)}\right) \frac{\partial^{2} \phi_{d l}(x, t)}{\partial x^{2}}-\frac{a_{s} F}{a C} j(\eta),
\end{aligned}
$$

gives the same current/voltage response. The boundary conditions of this system are given in Table IV, which also have the logarithmic term eliminated.

In effect, Assumptions 1 and 3 have removed the effect of the electrolyte concentration from the DFN model. Simulations results in $[24,23]$ suggest that the electrolyte polarisation will only be noticeable under high C-rate (>1-C) charging and it is predicted that it will also be important in dynamic charging at frequencies $\approx 1 \mathrm{rad}$ $\mathrm{s}^{-1}$ (as this corresponds to the time constant of the electrolyte). However, further studies are needed to fully undertstand the contribution of the electrolyte flux to the battery dynamics. The difficulty with including the electroltye within an observer is that $c_{e}$ couples the various dynamics together and in doing so complicates the stbility analysis. For this reason, the only observers that have been develloped with electrolyte effects included are either open loop [24] or require unphysical measurements [23].

The two state system (17) is used in the following section for the observer design. Even though the following observer is designed for this simpler system, the simulations of Section V are compared against the full DFN model with double layer effects included.

\section{OBserver Design}

A Luenberger observer for the battery model described in the previous section is developed here. Using the observer to obtain more accurate estimates of the model states $\left(u_{s}, \phi_{d l}\right)$ results in improved estimates of the $S o C$, defined here following [25] as

$$
\operatorname{SoC}(x, t)=\frac{3}{c_{s, \max }} \int_{0}^{R_{s}} u_{s}(x, r, t) d r
$$




\begin{tabular}{|c|c|}
\hline \multicolumn{2}{|c|}{ Boundary conditions of the observer } \\
\hline \multicolumn{2}{|c|}{ Double-layer potential } \\
\hline Current collector/anode & Anode/separator \\
\hline$\frac{\partial \hat{\phi}_{d l}}{\partial x}=-\frac{i(t)}{\sigma_{1}^{\text {eff }}}-l\left(\frac{\kappa_{1}^{\text {eff }}}{\kappa_{1}^{\text {eff }}+\sigma_{1}^{\text {eff }}}\right) \tilde{v}(t)$ & $\frac{\partial \hat{\phi}_{d l}}{\partial x}=\frac{i(t)}{\kappa_{1}^{\text {eff }}}-l\left(\frac{\sigma_{1}^{\text {eff }}}{\kappa_{1}^{\text {eff }}+\sigma_{1}^{\text {eff }}}\right) \tilde{v}(t)$ \\
\hline Separator/cathode & Cathode/current collector \\
\hline$\frac{\partial \hat{\phi}_{d l}}{\partial x}=\frac{i(t)}{\kappa_{3}^{\text {eff }}}+l\left(\frac{\sigma_{3}^{\text {eff }}}{\kappa_{3}^{\text {eff }}+\sigma_{3}^{\text {eff }}}\right) \tilde{v}(t)$ & $\frac{\partial \hat{\phi}_{d l}}{\partial x}=-\frac{i(t)}{\sigma_{3}^{\text {eff }}}+l\left(\frac{\kappa_{3}^{\text {eff }}}{\kappa_{3}^{\text {eff }}+\sigma_{3}^{\text {eff }}}\right) \tilde{v}(t)$ \\
\hline \multicolumn{2}{|c|}{ Ionic concentration in the particles } \\
\hline Particle centre & Particle surface \\
\hline$\hat{u}_{s}(0, t)=0$ & $\left.\frac{1}{R_{s}} \frac{\partial \hat{u}_{s}}{\partial r}\right|_{r=R_{s}}-\frac{\hat{u}_{s}\left(x, R_{s}, t\right)}{R_{s}^{2}}=\frac{-j(\hat{\eta})}{D_{s}}$ \\
\hline
\end{tabular}

Table V: Boundary conditions of the observer states. The only difference between the observer and the reduced DFN model is the output injection term entering into the boundary conditions on $\hat{\phi}_{d l}$. The observer gain $l$ is a design parameter.

with $c_{s, \max }$ being the maximum particle concentration. This expression allows the bulk state-of-charge for the anode $\overline{S o C}$ to be defined

$$
\overline{S o C}(t)=\frac{1}{L_{12}} \int_{0}^{L_{12}} \operatorname{SoC}(x, t) d x .
$$

A similar bulk state-of-charge could be obtained for the cathode by integrating from $x=L_{23}$ to $x=L_{\text {bat }}$. Information on the spatial distribution of the overpotential can also be obtained, which is essential for avoiding lithium plating [31]. The observer develops upon the results of [2] and [15, 5] for finite dimensional systems (also with monotonic nonlinearities) to the considered class of parabolic PDEs. Such a passivity based approach allows the nonlinearities of the model to be considered, unlike the backstepping approach of $[40,20]$. The plant considered in this section is the reduced order system (17) with the boundary conditions of Table IV.

$\psi\left(y_{j}\right)$

$y_{j}$

$k_{j} y_{j}$

The observer for estimating the state of (17) has dynamics

$$
\begin{aligned}
\frac{\partial \hat{u}_{s}(r, t)}{\partial t} & =D_{s} \frac{\partial^{2} \hat{u}_{s}(r, t)}{\partial r^{2}}, \\
\frac{\partial \hat{\phi}_{d l}(x, t)}{\partial t} & =\left(\frac{\kappa^{\text {eff }} \sigma^{\text {eff }}}{a C\left(\sigma^{\text {eff }}+\kappa^{\text {eff }}\right)}\right) \frac{\partial^{2} \hat{\phi}_{d l},(x, t)}{\partial x^{2}}-\frac{a_{s} F}{a C} j(\hat{\eta})
\end{aligned}
$$

with the observer voltage being

$$
\begin{aligned}
\hat{v}(t)= & -\left(\frac{\kappa_{1}^{\mathrm{eff}}}{\kappa_{1}^{\mathrm{eff}}+\sigma_{1}^{\mathrm{eff}}}\right) \hat{\phi}_{d l}(0, t)-\left(\frac{\sigma_{1}^{\mathrm{eff}}}{\kappa_{1}^{\mathrm{eff}}+\sigma_{1}^{\mathrm{eff}}}\right) \hat{\phi}_{d l}\left(L_{12}, t\right) \\
& +\left(\frac{\sigma_{3}^{\mathrm{eff}}}{\kappa_{3}^{\mathrm{eff}}+\sigma_{3}^{\mathrm{eff}}}\right) \hat{\phi}_{d l}\left(L_{23}, t\right)+\left(\frac{\kappa_{3}^{\mathrm{eff}}}{\kappa_{3}^{\mathrm{eff}}+\sigma_{3}^{\mathrm{eff}}}\right) \hat{\phi}_{d l}\left(L_{\mathrm{bat}}, t\right) \\
& -\left(\frac{1}{\kappa_{1}^{\mathrm{eff}}\left(\kappa_{1}^{\mathrm{eff}}+\sigma_{1}^{\mathrm{eff}}\right)}+\frac{1}{\kappa_{3}^{\mathrm{eff}}\left(\kappa_{3}^{\mathrm{eff}}+\sigma_{3}^{\mathrm{eff}}\right)}+\frac{1}{\kappa_{2}^{\mathrm{eff}}}-R_{\mathrm{ctc}}\right) i
\end{aligned}
$$

The observer states are subject to the boundary conditions of Table $\mathrm{V}$ with $l$ being the observer gain. The only difference between the observer and the battery model is the output injection term entering into the boundary conditions of $\hat{\phi}(x, t)$, which was designed by considering the definition of the voltage.

Of fundamental importance to the performance of any observer is the model's underlying observability. The authors are not aware of any complete tests for checking the observability of nonlinear infinite dimensional systems. There are two methods to check the approximate observability of this system. The first involves linearising (16)(17) and then applying the approximate observability test of [8, Chapter 4]. The second discretises the nonlinear PDEs in space and then applies the observability test for nonlinear finite dimensional systems from [16]. Both of these methods have limitations; the mapping from output to state of the linearised system is not equivalent to that of the the nonlinear system and the nonlinear observability test of [16] involves computing Lie derivatives along the state trajectories which is not scalable for systems with $\gg 10$ states, as encountered here with the spatially discretised model (17). Such a nonlinear observability test was applied to a spatially discretised electrochemical supercapcitor model in [12] and to an equivalent circuit model in [47], with both of these studies emphasising discrepancies between the nonlinear and linear analysis.

The following convergence result for the observer error is obtained.

Theorem 1: If the particle surface concentration error is sufficiently small and the observer gain $l$ is non-negative, then the observer error is asymptotically stable.

Proof. Define the error system, with $\tilde{u}_{s}=u_{s}-\hat{u}_{s}, \tilde{\phi}_{d l}=$ $\phi_{d l}-\hat{\phi}_{d l}$ and $\tilde{v}(t)=v(t)-\hat{v}(t)$,

$$
\begin{aligned}
\frac{\partial \tilde{u}_{s}}{\partial t} & =D_{s} \frac{\partial^{2} \tilde{u}_{s}}{\partial r^{2}} \\
\frac{\partial \tilde{\phi}_{d l}}{\partial t} & =\left(\frac{\kappa^{\mathrm{eff}} \sigma^{\mathrm{eff}}}{a C\left(\sigma^{\mathrm{eff}}+\kappa^{\mathrm{eff}}\right)}\right) \frac{\partial^{2} \tilde{\phi}_{d l}}{\partial x^{2}}-\frac{a_{s} F}{a C}(j(\hat{\eta})-j(\eta)) .
\end{aligned}
$$$$
i(t)
$$

Convergence of the observer state towards that of the plant follows from the asymptotic stability of this error system. 
Consider the Lyapunov functional

$$
\begin{aligned}
V(t) & =\left\|\tilde{u}_{s}\right\|_{\mathcal{L}_{2}\left(\Omega_{x} \times \Omega_{r}\right)}^{2}+\left\|\tilde{\phi}_{d l}\right\|_{\mathcal{L}_{2}\left(\Omega_{x}\right)}^{2} \\
& =\frac{1}{2} \int_{\Omega_{x}} \int_{\Omega_{r}} \tilde{u}_{s}(x, r, t)^{2} d r+\tilde{\phi}_{d l}(x, t)^{2} d x
\end{aligned}
$$

Differentiating $V(t)$ along the vector field of the error system (22) gives

$$
\begin{aligned}
\dot{V}(t) & =\int_{\Omega_{x}} \int_{\Omega_{r}} \tilde{u}_{s} \dot{\tilde{u}}_{s} d r+\tilde{\phi}_{d l} \dot{\tilde{\phi}}_{d l} d x \\
& =\int_{\Omega_{x}} \int_{\Omega_{r}} \tilde{u}_{s}\left(D_{s} \frac{\partial^{2} \tilde{u}_{s}}{\partial r^{2}}\right) d r
\end{aligned}
$$

$+\tilde{\phi}_{d l}\left(\left(\frac{\kappa^{\mathrm{eff}} \sigma^{\mathrm{eff}}}{a C\left(\sigma^{\mathrm{eff}}+\kappa^{\mathrm{eff}}\right)}\right) \frac{\partial^{2} \tilde{\phi}_{d l}}{\partial x^{2}}-\frac{a_{s} F}{a C}(j(\hat{\eta})-j(\eta))\right) d x$

Applying integration by parts, and using the boundary conditions of $\phi_{d l}(x, t), \hat{\phi}_{d l}(x, t), u_{s}(x, r, t)$, and $\hat{u}_{s}(x, r, t)$ from Tables IV and $\mathrm{V}$ gives

$$
\begin{aligned}
\dot{V}(t) & =\left.\tilde{\phi}_{d l} \frac{\partial \tilde{\phi}_{d l}}{\partial x}\right|_{x=0} ^{x=L_{12}}+\left.\tilde{\phi}_{d l} \frac{\partial \tilde{\phi}_{d l}}{\partial x}\right|_{x=L_{23}} ^{x=L} \\
& +\int_{\Omega_{x}} D_{s} \frac{\left(\tilde{u}_{s}^{\text {surf }}\right)^{2}}{R}+\int_{\Omega_{r}}-D_{s}\left(\frac{\partial \tilde{u}_{s}}{\partial r}\right)^{2} d r \\
& -\left(\frac{\kappa^{\mathrm{eff}} \sigma^{\mathrm{eff}}}{a C\left(\sigma^{\mathrm{eff}}+\kappa^{\mathrm{eff}}\right)}\right)\left(\frac{\partial \tilde{\phi}_{d l}}{\partial x}\right)^{2} \\
& -\frac{a_{s} F}{a C}(j(\hat{\eta})-j(\eta))\left(\tilde{\phi}_{d l}+\frac{R_{s} a C}{a_{s} F} \tilde{u}_{s}^{\text {surf }}\right) d x .
\end{aligned}
$$

Under the assumption that the surface concentration error is small, then the last term in (25) is approximately

$$
\begin{aligned}
& (j(\hat{\eta})-j(\eta))\left(\tilde{\phi}_{d l}+\frac{R_{s} a C}{a_{s} F} \tilde{u}_{s}^{\text {surf }}\right) \\
& =(j(\hat{\eta})-j(\eta))(\hat{\eta}-\eta) \\
& \quad+(j(\hat{\eta})-j(\eta))\left(U\left(u_{s}^{\text {surf }}\right)-U\left(\hat{u}_{s}^{\text {surf }}\right)+\frac{R_{s} a C}{D_{s} a_{s} F} \tilde{u}_{s}^{\text {surf }}\right), \\
& \approx(j(\hat{\eta})-j(\eta))(\hat{\eta}-\eta),
\end{aligned}
$$

which is non-negative due to the monotonicity of the Butler-Volmer kinetics when $i_{0}$ is positive and constant

$$
(j(\hat{\eta})-j(\eta))(\hat{\eta}-\eta) \geq 0
$$

for all $\eta$ and $\hat{\eta}$. The bound

$$
\begin{aligned}
\dot{V}(t) \leq & \left.\tilde{\phi}_{d l} \frac{\partial \tilde{\phi}_{d l}}{\partial x}\right|_{x=0} ^{x=L_{12}}+\left.\tilde{\phi}_{d l} \frac{\partial \tilde{\phi}_{d l}}{\partial x}\right|_{x=L_{23}} ^{x=L} \\
& +\int_{\Omega_{x}} D_{s} \frac{\left(\tilde{u}_{s}^{\text {surf }}\right)^{2}}{R}+\int_{\Omega_{r}}-D_{s}\left(\frac{\partial \tilde{u}_{s}}{\partial r}\right)^{2} d r d x
\end{aligned}
$$

is then obtained.

Since $u_{s}(x, 0, t)=\hat{u}_{s}(x, 0, t)=\tilde{u}_{s}(x, 0, t)=0$, then

$$
\left(\tilde{u}_{s}^{\text {surf }}\right)^{2}=\left(\int_{\Omega_{r}} \frac{\partial \tilde{u}_{s}}{\partial r} d r\right)^{2}
$$

hence

$$
\begin{aligned}
& \frac{\left(\tilde{u}^{\text {surf }}\right)^{2}}{R}-\int_{\Omega_{r}}\left(\frac{\partial \tilde{u}_{s}}{\partial r}\right)^{2} d r \\
& =\frac{1}{R}\left(\int_{\Omega_{r}} \frac{\partial \tilde{u}_{s}}{\partial r} d r\right)^{2}-\int_{\Omega_{r}}\left(\frac{\partial \tilde{u}_{s}}{\partial r}\right)^{2} d r
\end{aligned}
$$

By first normalising $r \in \Omega_{r}=\left(0, R_{s}\right)$ to $\hat{r} \in(0,1)$

$$
\begin{aligned}
& \frac{1}{R}\left(\int_{\Omega_{r}} \frac{\partial \tilde{u}_{s}}{\partial r} d r\right)^{2}-\int_{\Omega_{r}}\left(\frac{\partial \tilde{u}_{s}}{\partial r}\right)^{2} d r \\
& =\frac{1}{R}\left(\left(\int_{0}^{1} \frac{\partial \tilde{u}_{s}}{\partial \hat{r}} d \hat{r}\right)^{2}-\int_{0}^{1}\left(\frac{\partial \tilde{u}_{s}}{\partial \hat{r}}\right)^{2} d \hat{r}\right)
\end{aligned}
$$

and then applying the Cauchy-Schwartz inequality, it follows that

$$
\left(u_{s}^{\text {surf }}\right)^{2}=\left(\int_{0}^{1} \frac{\partial \tilde{u}_{s}}{\partial \hat{r}} d \hat{r}\right)^{2} \leq \int_{0}^{1}\left(\frac{\partial \tilde{u}_{s}}{\partial \hat{r}}\right)^{2} d \hat{r}
$$

giving

$$
\dot{V}(t) \leq\left.\tilde{\phi} \frac{\partial \tilde{\phi}}{\partial x}\right|_{x=0} ^{x=L_{12}}+\left.\tilde{\phi} \frac{\partial \tilde{\phi}}{\partial x}\right|_{x=L_{23}} ^{x=L} .
$$

The judicious choice of boundary conditions on $\hat{\phi}_{d l}$, and hence $\tilde{\phi}_{d l}$, means the growth rate of $V(t)$ is bounded from above by the negative of the "energy" of the voltage error

$$
\begin{aligned}
\dot{V}(t) \leq & l \tilde{v}(t)\left(\left(\frac{\kappa_{1}^{\mathrm{eff}}}{\kappa_{1}^{\mathrm{eff}}+\sigma_{1}^{\mathrm{eff}}}\right) \tilde{\phi}_{d l}(0, t)+\left(\frac{\sigma_{1}^{\mathrm{eff}}}{\kappa_{1}^{\mathrm{eff}}+\sigma_{1}^{\mathrm{eff}}}\right) \tilde{\phi}_{d l}\left(L_{12}, t\right)\right. \\
& \left.-\left(\frac{\sigma_{3}^{\mathrm{eff}}}{\kappa_{3}^{\mathrm{eff}}+\sigma_{3}^{\mathrm{eff}}}\right) \tilde{\phi}_{d l}\left(L_{23}, t\right)-\left(\frac{\kappa_{3}^{\mathrm{eff}}}{\kappa_{3}^{\mathrm{eff}}+\sigma_{3}^{\mathrm{eff}}}\right) \tilde{\phi}_{d l}\left(L_{\mathrm{bat}}, t\right)\right), \\
= & -l \tilde{v}(t)^{2},
\end{aligned}
$$

and since $-l \tilde{v}(t)^{2} \leq 0$, the observer error is asymptotically stable.

\section{Finite Dimensional Algorithmic Search}

An algorithmic method to compute gains for the observer (20) can be obtained by first spatially discretising the system and then applying Theorem 1 of [2]. The resulting observer gains could then be constructed from the solution of a linear matrix inequality (LMI) which in principle can be efficiently solved using convex programming.

However, this finite dimensional approach suffers from two main limitations when applied to the battery model. The first relates to the size of the discretised system, with roughly 100 states being needed for the discretised battery model. Current LMI solvers often struggle to converge to a suitable tolerance for systems of this size. This issue is exacerbated by the fact that the DFN model dynamics contain time scales that are separated by orders of magnitude (with the relaxation of the double layer being significantly faster than lithium intercalation into the particles). This means that if solutions to the LMI can be found, then they will most likely be close to rank deficient. As such it will not be possible to obtain the 
observer gains by matrix inversion [2, Theorem 1]. The second issue is that since batteries are energy storage devices, they contain integrators and dynamic modes that are lossless [13]. These modes would not be in $\mathcal{L}_{2}$, implying that methods such as encapsulation [18] or loop transformations [19] may have to be applied. It is noted that the decomposition constructed in [13] for supercapacitors could not be applied to this battery model. It was for these reasons that the observer design based on a Luenberger observer was applied to the PDEs of the model.

\section{Simulations}

For implementation purposes, the observer system was discretised in space using the spectral collocation method [43] developed in [4] for the battery model. By taking advantage of the spectral convergence rates of this discretisation scheme, relatively few ( $\sim 10$ in each sub-domain) grid points were needed to achieve a sufficient level of solution accuracy. The spatially discretised systems were coded in MATLAB and were simulated using the ode15s ordinary differential equation solver [38]. The model parameter values used in the simulations were obtained from [4] except for the specific capacitance which was taken to be $a C=22 \times 10^{3} F^{-2}$ and the ion exchange current densities of the observer, with $i_{0}=0.706$ in the observer's anode and $i_{0}=5.231$ in its cathode.

Figures 1 and 2 show the results of simulations of the observer, with Figure 1 being applied with a $1 \mathrm{C}$ constant current discharge with $i=20 \mathrm{~A} \mathrm{~m}^{-2}$ whilst the current of Figure 2 was obtained from the ARTEMIS drive cycle [1]. The observer gain for both simulations was set to $l=$ $10^{4}$. In order to guarantee well-posedness of the PDEs, the initial conditions of the observer were set to be the state of the plant at $t=1000 \mathrm{~s}$ from the simulation of the constant current discharge, with the plant being battery model described by (17). For both of these simulations, the ion exchange current density of the plant was spatially varying, as in it satisfied (4), although the value of $i_{0}$ used in the observer was set to a constant value, as discussed above.

The observer was found to perform well for these charge profiles and in fact, exponential convergence of the observer error was achieved even with the simplified model of the ion exchange density. This suggests that the observer exhibits both a degree of robustness and a high level of performance. Furthermore, a monotonic decrease in the error of $u_{s}$ was achieved, which is particularly useful as this is the spatio-temporal variable of interest for $S o C$ estimation. The restriction on $i_{0}$ to be constant in the observer was evaluated in Figure 3, with this simulation again being applied with the constant current discharge except with $i_{0}$ now being constant also for the plant. This figure contrasts with that of Figure 1e, with there now being a more monotonic decrease in the error, however, surprisingly, this decrease was not as rapid as for the spatially varying $i_{0}$ case.

The effect of varying the observer gain $l$ is considered in Figure 4, where simulations for the constant current discharge are shown for different values of $l$. It was found that increasing the gain led to faster convergence although increasing the gain will also amplify noise in the sensor measurements.

\section{Conclusions}

An electrochemical model and an observer were developed for the problem of state-of-charge estimation of a Li-ion battery model. The model was derived from the Doyle-Fuller-Newman model that included double layer effects and assumed that MacInnes' equation was independent of the ionic concentration and the ion exchange current density was constant. The observer was designed for the resulting non-linear partial differential equations by exploiting the monotonicity of the Butler-Volmer reaction kinetics. It was shown that the observers were able to achieve exponential convergence in their estimate of the state whilst also being relatively robust. This result enables observer design based on this more electrochemically justifiable case providing more accurate estimates of the battery's state-of-charge when used within a battery management system.

\section{REFERENCES}

[1] M. André, "The ARTEMIS European driving cycles for measuring car pollutant emissions," Science of the total Environment, vol. 334, pp. 73-84, 2004.

[2] M. Arcak and P. Kokotović, "Nonlinear observers: A circle criterion design and robustness analysis," Automatica, vol. 37, no. 12, pp. 1923-1930, 2001.

[3] P. Ascencio, A. Astolfi, and T. Parisini, "Backstepping PDE-based adaptive observer for a single particle model of lithium-ion batteries," in Proc. of the IEEE Conference on Decision and Control. IEEE, 2016, pp. 5623-5628.

[4] A. M. Bizeray, S. Zhao, S. R. Duncan, and D. A. Howey, "Lithium-ion battery thermal-electrochemical model-based state estimation using orthogonal collocation and a modified extended Kalman filter," Journal of Power Sources, vol. 296, pp. 400-412, 2015.

[5] A. Chakrabarty, M. Corless, G. T. Buzzard, S. H. Zak, and A. E. Rundell, "State and unknown input observers for nonlinear systems with bounded exogenous inputs," IEEE Transactions on Automatic Control, 2017.

[6] H. Chaoui, N. Golbon, I. Hmouz, R. Souissi, and S. Tahar, "Lyapunov-based adaptive state of charge and state of health estimation for lithium-ion batteries," IEEE Transactions on Industrial Electronics, vol. 62, no. 3, pp. 1610-1618, 2015.

[7] M. Corno, N. Bhatt, S. M. Savaresi, and M. Verhaegen, "Electrochemical model-based state of charge estimation for Li-ion cells," IEEE Transactions on Control Systems Technology, vol. 23, no. 1, pp. 117127, 2015.

[8] R. F. Curtain and H. Zwart, An Introduction to Infinite-dimensional Linear Systems Theory. Springer Science \& Business Media, 2012, vol. 21. 


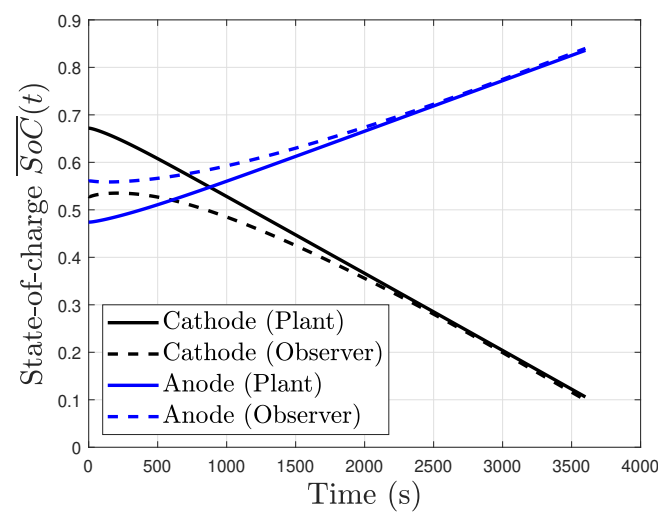

(a) State-of-charge.

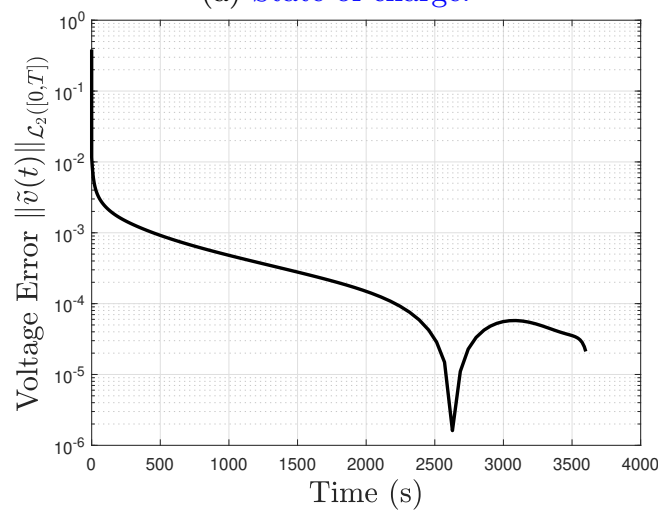

(c) Voltage error.

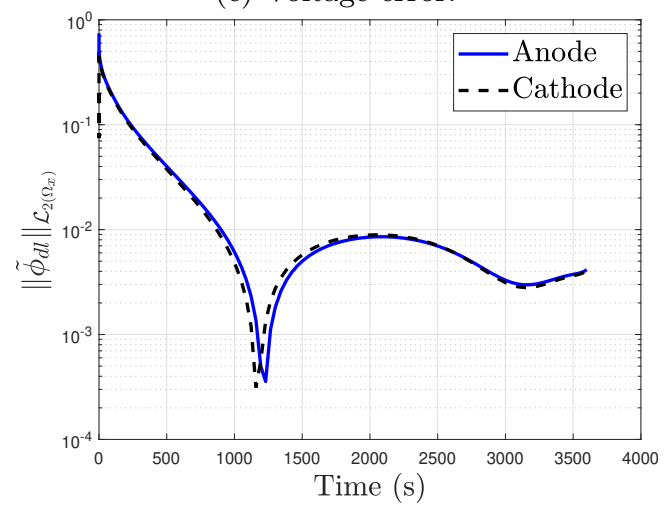

(e) Error norm of ionic concentration in particles.

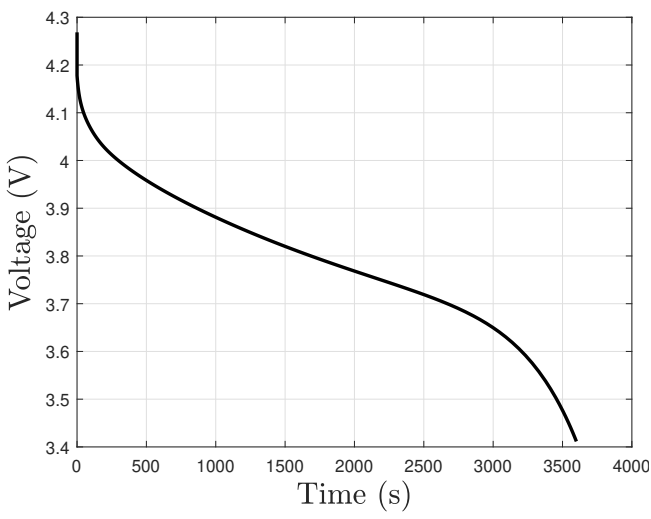

(b) Plant voltage.

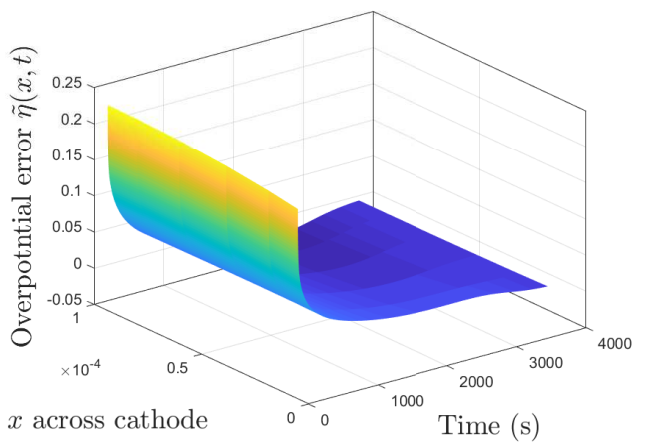

(d) Overpotential error across the cathode.

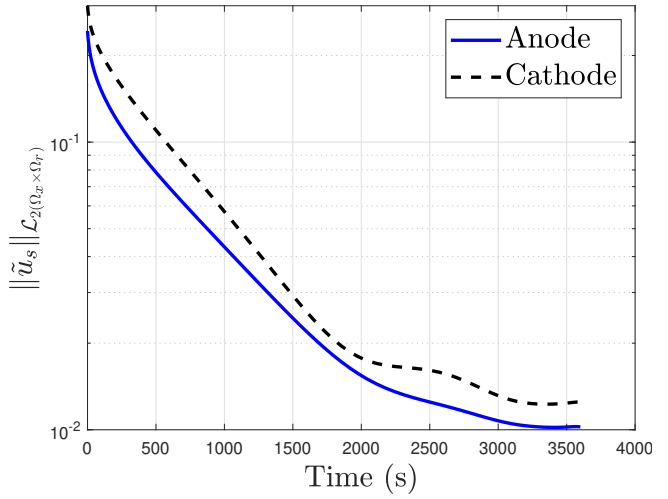

(f) Error norm of the electrode potential.

Figure 1: Response of the observer under the discharging current of the ARTEMIS drive cycle.

[9] S. Dey, B. Ayalew, and P. Pisu, "Nonlinear robust observers for state-of-charge estimation of lithiumion cells based on a reduced electrochemical model," IEEE Transactions on Control Systems Technology, vol. 23, no. 5, pp. 1935-1942, 2015.

[10] M. Doyle, T. F. Fuller, and J. Newman, "Modeling of galvanostatic charge and discharge of the lithium/polymer/insertion cell," Journal of the Electrochemical Society, vol. 140, no. 6, pp. 1526-1533, 1993.

[11] R. Drummond, A. Bizeray, and S. Duncan, "A feedback interpretation of the Doyle-Fuller-Newman Liion battery model," Under review at IEEE Transactions on Control System Technology.
[12] R. Drummond and S. R. Duncan, "On observer performance for an electrochemical supercapacitor model." in IFAC Multi-Conference on Systems and Control, Sydney, AUS, 2015, pp. 1260-1265.

[13] R. Drummond, S. Zhao, and S. Duncan, "Design tools for electrochemical supercapacitors using local absolute stability theory," To be published in IEEE Transactions on Control System Technology.

[14] R. Drummond, S. Zhao, D. A. Howey, and S. R. Duncan, "Circuit synthesis of electrochemical supercapacitor models," Journal of Energy Storage, vol. 10, pp. 48-55, 2017.

[15] Q. P. Ha and H. Trinh, "State and input simultaneous estimation for a class of nonlinear systems," Automat- 


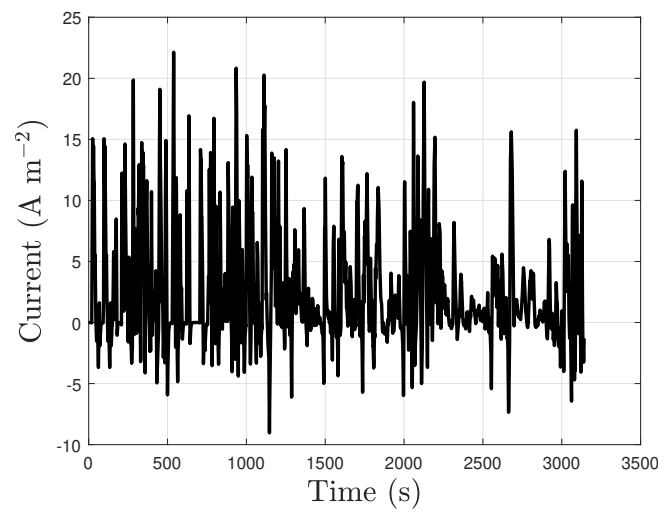

(a) Applied current.

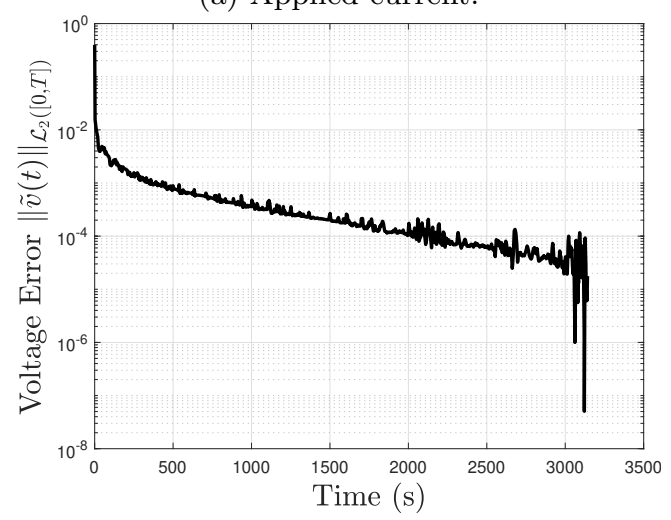

(c) Voltage error.

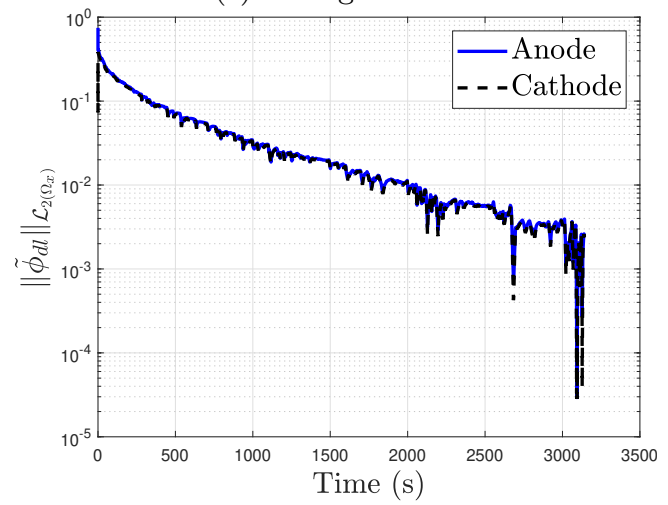

(e) Error norm of ionic concentration in particles.

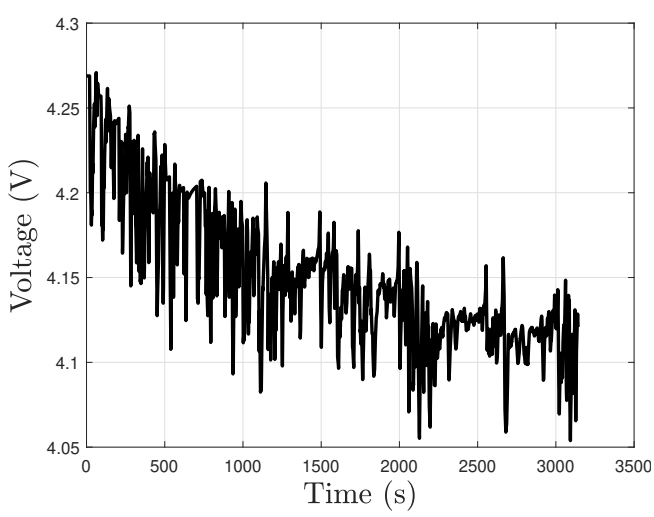

(b) Plant voltage.

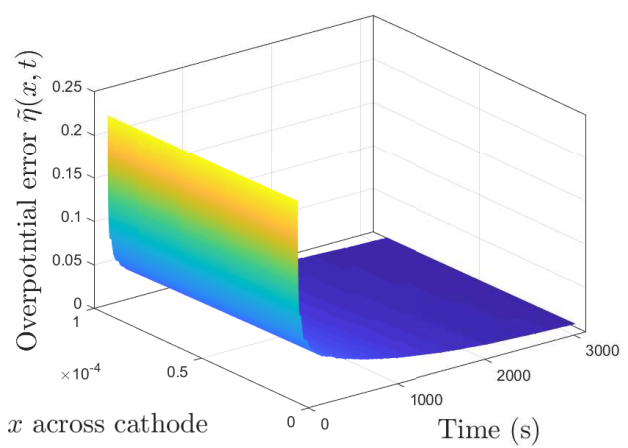

(d) Overpotential error across the cathode.

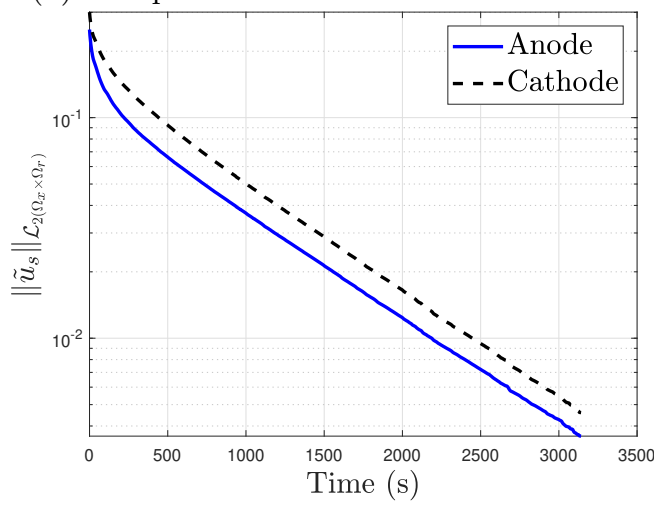

(f) Error norm of the electrode potential.

Figure 2: Response of the observer under the charging current of the ARTEMIS drive cycle.

ica, vol. 40, no. 10, pp. 1779-1785, 2004.

[16] R. Hermann and A. Krener, "Nonlinear controllability and observability," IEEE Transactions on automatic control, vol. 22, no. 5, pp. 728-740, 1977.

[17] X. Hu, S. Li, and H. Peng, "A comparative study of equivalent circuit models for Li-ion batteries," Journal of Power Sources, vol. 198, pp. 359-367, 2012.

[18] U. Jonsson and A. Megretski, "The Zames-Falb IQC for critically stable systems," in Proc. of the American Control Conference, vol. 6. IEEE, 1998, pp. 36123616.

[19] H. K. Khalil, Nonlinear systems. Upper Saddle River, NJ, USA: Prentice-Hall, 1996, vol. 16.

[20] M. Krstic and A. Smyshlyaev, Boundary control of
PDEs: A course on backstepping designs. Philadelphia, PA, USA: SIAM, 2008, vol. 16.

[21] Z. Li, J. Huang, B. Y. Liaw, and J. Zhang, "On state-of-charge determination for lithium-ion batteries," Journal of Power Sources, vol. 348, pp. 281-301, 2017.

[22] W. Luo, C. Lyu, L. Wang, and L. Zhang, "A new extension of physics-based single particle model for higher charge-discharge rates," Journal of Power Sources, vol. 241, pp. 295-310, 2013.

[23] P.-F. Massiani, R. Drummond, D. Zhang, and S. Moura, "An infinite-dimensional state observer for a Doyle-Fuller-Newman battery model with double layer capacitance dynamics." in Submitted to the IEEE 


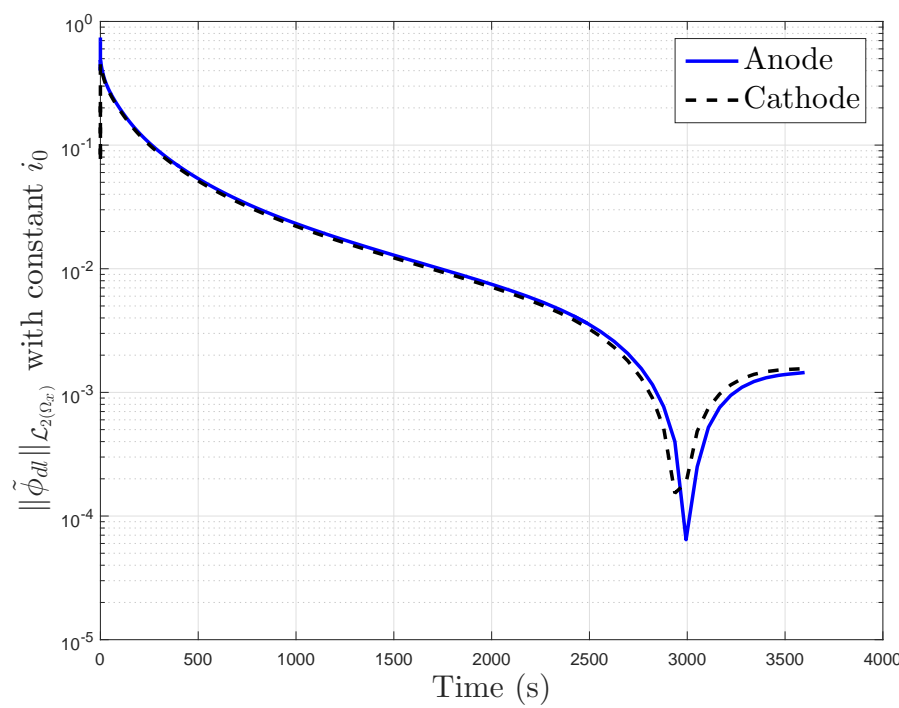

Figure 3: Error norm of the electrode potential for the $1 \mathrm{C}$ constant current discharge with $i_{0}$ of the plant kept constant.

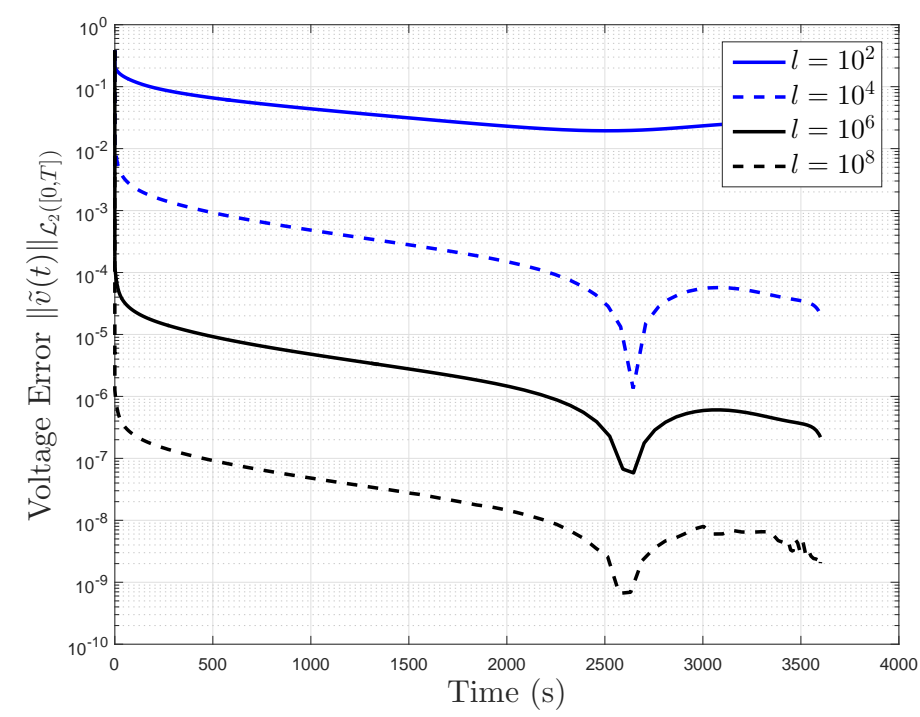

Figure 4: Voltage error under a $1 \mathrm{C}$ constant current discharge with different observer gains $l$.

American Control Conference, 2019.

[24] S. J. Moura, F. B. Argomedo, R. Klein, A. Mirtabatabaei, and M. Krstic, "Battery state estimation for a single particle model with electrolyte dynamics," IEEE Transactions on Control Systems Technology, vol. 25, no. 2, pp. 453-468, 2017.

[25] S. J. Moura, N. A. Chaturvedi, and M. Krstic, "PDE estimation techniques for advanced battery management systems-Part I: SOC estimation," in Proc of the American Control Conference. IEEE, 2012, pp. 559565.

[26] S. J. Moura, N. A. Chaturvedi, and M. Krstić, "Adaptive partial differential equation observer for battery state-of-charge/state-of-health estimation via an electrochemical model," Journal of Dynamic Systems,
Measurement, and Control, vol. 136, no. 1, p. 011015 , 2014.

[27] J. Newman and K. E. Thomas-Alyea, Electrochemical systems. John Wiley \& Sons, 2012.

[28] G. Ning and B. N. Popov, "Cycle life modeling of lithium-ion batteries," Journal of The Electrochemical Society, vol. 151, no. 10, pp. A1584-A1591, 2004.

[29] K.-Y. Oh, B. I. Epureanu, J. B. Siegel, and A. G. Stefanopoulou, "Phenomenological force and swelling models for rechargeable lithium-ion battery cells," Journal of Power Sources, vol. 310, pp. 118-129, 2016.

[30] I. J. Ong and J. Newman, "Double-layer capacitance in a dual lithium ion insertion cell," Journal of The Electrochemical Society, vol. 146, no. 12, pp. 4360 4365, 1999.

[31] H. Perez, N. Shahmohammadhamedani, and S. Moura, "Enhanced performance of li-ion batteries via modified reference governors and electrochemical models," IEEE/ASME Transactions on Mechatronics, vol. 20, no. 4, pp. 1511-1520, 2015.

[32] G. L. Plett, "Extended Kalman filtering for battery management systems of LiPB-based HEV battery packs: Part 3. State and parameter estimation," Journal of Power sources, vol. 134, no. 2, pp. 277-292, 2004.

[33] — , Battery Management Systems, Volume I: Battery Modeling. Artech House, 2015, vol. 1.

[34] V. Ramadesigan, P. W. Northrop, S. De, S. Santhanagopalan, R. D. Braatz, and V. R. Subramanian, "Modeling and simulation of lithium-ion batteries from a systems engineering perspective," Journal of The Electrochemical Society, vol. 159, no. 3, pp. R31R45, 2012.

[35] R. Rao, S. Vrudhula, and D. N. Rakhmatov, "Battery modeling for energy aware system design," Computer, vol. 36, no. 12, pp. 77-87, 2003.

[36] N. A. Samad, Y. Kim, J. B. Siegel, and A. G. Stefanopoulou, "Battery capacity fading estimation using a force-based incremental capacity analysis," Journal of The Electrochemical Society, vol. 163, no. 8, pp. A1584-A1594, 2016.

[37] S. Santhanagopalan, Q. Guo, P. Ramadass, and R. E. White, "Review of models for predicting the cycling performance of lithium ion batteries," Journal of Power Sources, vol. 156, no. 2, pp. 620-628, 2006.

[38] L. F. Shampine and M. W. Reichelt, "The matlab ode suite," SIAM journal on scientific computing, vol. 18, no. 1, pp. 1-22, 1997.

[39] K. A. Smith, C. D. Rahn, and C.-Y. Wang, "Control oriented 1D electrochemical model of lithium ion battery," Energy Conversion and management, vol. 48, no. 9, pp. 2565-2578, 2007.

[40] A. Smyshlyaev and M. Krstic, "Backstepping observers for a class of parabolic PDEs," Systems \&6 Control Letters, vol. 54, no. 7, pp. 613-625, 2005.

[41] S.-X. Tang, L. Camacho-Solorio, Y. Wang, and M. Krstic, "State-of-charge estimation from a thermal-electrochemical model of lithium-ion batter- 
ies," Automatica, vol. 83, pp. 206-219, 2017.

[42] K. E. Thomas, J. Newman, and R. M. Darling, "Mathematical modeling of lithium batteries," in Advances in lithium-ion batteries. Springer, 2002, pp. 345-392.

[43] L. N. Trefethen, Spectral methods in MATLAB. Philadelphia, PA, USA: SIAM, 2000.

[44] M. W. Verbrugge and P. Liu, "Microstructural analysis and mathematical modeling of electric doublelayer supercapacitors," Journal of the Electrochemical Society, vol. 152, no. 5, pp. D79-D87, 2005.

[45] M.-T. von Srbik, M. Marinescu, R. F. MartinezBotas, and G. J. Offer, "A physically meaningful equivalent circuit network model of a lithium-ion battery accounting for local electrochemical and thermal behaviour, variable double layer capacitance and degradation," Journal of Power Sources, vol. 325, pp. 171-184, 2016.

[46] W. Waag, C. Fleischer, and D. U. Sauer, "Critical review of the methods for monitoring of lithium-ion batteries in electric and hybrid vehicles," Journal of Power Sources, vol. 258, pp. 321-339, 2014.

[47] S. Zhao, S. R. Duncan, and D. A. Howey, "Observability analysis and state estimation of lithiumion batteries in the presence of sensor biases," IEEE Transactions on Control Systems Technology, vol. 25, no. 1, pp. 326-333, 2017.

[48] C. Zou, C. Manzie, D. Nešić, and A. G. Kallapur, "Multi-time-scale observer design for state-of-charge and state-of-health of a lithium-ion battery," Journal of Power Sources, vol. 335, pp. 121-130, 2016. 